\title{
The effect of gender on response to antithyroid drugs and risk of relapse after discontinuation of the antithyroid drugs in patients with Graves' hyperthyroidism: a multicentre study
}

\author{
Sayid Shafi Zuhur', Ismail Yildiz', Yuksel Altuntas², Taner Bayraktaroglü ${ }^{3}$, Selvinaz Erol'2, Serdar Sahin ${ }^{4}$, \\ Faruk Kilinc ${ }^{5}$, Gulhan Akbaba ${ }^{6}$, Birol Topcu ${ }^{7}$, Gulsah Elbuken ${ }^{1}$, Pinar Kadioglu ${ }^{4}$ \\ ${ }^{1}$ Department of Endocrinology and Metabolism, Faculty of Medicine, Namik Kemal University, Tekirdag, Turkey \\ ${ }^{2}$ Department of Endocrinology and Metabolism, Health Science University, Sisli Hamidiye Etfal Training and Research Hospital, \\ Istanbul, Turkey \\ ${ }^{3}$ Department of Endocrinology and Metabolism, Faculty of Medicine, Bulent Ecevit University, Zonguldak, Turkey \\ ${ }^{4}$ Department of Endocrinology and Metabolism, Cerrahpasa Faculty of Medicine, Istanbul University, Istanbul, Turkey \\ ${ }^{5}$ Department of Endocrinology and Metabolism, Faculty of Medicine, Firat University, Elazig, Turkey \\ ${ }^{6}$ Department of Endocrinology and Metabolism, Faculty of Medicine, Mugla Sitki Kocman University, Mugla, Turkey \\ ${ }^{7}$ Department of Biostatistics, Faculty of Medicine, Namik Kemal University, Tekirdag, Turkey
}

\begin{abstract}
Introduction: The outcome of medical treatment in patients with Graves' disease (GD) is generally difficult to predict. In this study, we examined the hypothesis that gender may affect the outcome of treatment with antithyroid drugs (ATDs).

Material and methods: This is a retrospective multicentre study including 717 (514 female and 203 male) patients with the first episode of GD treated for at least 12 months. Patients were classified as relapse, poorly controlled (several episodes of hyperthyroidism followed by euthyroidism and rarely hypothyroidism, occurring after titration of ATDs), and remission.

Results: During the mean follow-up time of $26.75 \pm 21.25$ months (between 1 and 120 months), 269 (37.5\%), $176(24.5 \%)$, and $272(37.9 \%)$ patients experienced a relapse, a poorly controlled disease, and remained in remission, respectively. During the follow-up time, 223 (43.4\%) of the female and only 49 (24\%) of the male patients remained in remission. Relapse and poorly controlled disease (non-remitting GD) were more common in male compared to female patients with GD (hazard ratio 1.26, 95\% CI: 1.03-1.53, $\mathrm{p}=0.025$ ). Graves' disease in male patients tended to relapse earlier, and male patients tended to have larger goiter sizes at diagnosis as well. The smoking habit was also significantly more frequent in males compared to female patients with GD.

Conclusion: Male patients with GD have a markedly higher frequency of relapse and poorly controlled disease, as compared to female patients. Larger goiter sizes and higher frequency of smoking may contribute to the higher frequency of relapse and poorly controlled disease in male patients. (Endokrynol Pol 2020; 71 (3): 207-212)
\end{abstract}

Key words: hyperthyroidism; Graves' disease; antithyroid drugs; gender

\section{Introduction}

Graves' disease (GD) is an autoimmune disorder and is the most common cause of hyperthyroidism in iodine-replete areas of the world, affecting women approximately 5-6 times more frequently than men [1]. According to epidemiological studies, about $3 \%$ of women and $0.5 \%$ of men develop GD during their lifetime [1]. Antithyroid drugs (ATDs), including methimazole and propylthiouracil, radioactive iodine treatment (RAI), and total thyroidectomy are the treatment options currently used for the treatment of GD. Although ATDs are the preferred treatment option, relapse is frequent and occurs in approximately $50 \%$ of patients after dis- continuation of ATDs [2]. Moreover, among patients treated with ATDs, a substantial number may experience several episodes of hyperthyroidism followed by euthyroidism and rarely hypothyroidism or vice versa, ensuing after titration of ATDs [3]. On the other hand, RAI treatment and total thyroidectomy usually result in permanent hypothyroidism that necessitates life-long levothyroxine treatment [3]. Therefore, to achieve a euthyroid state during treatment and long-term remission after discontinuation of the ATDs, appropriate selection of patients for treatment with ATDs is important.

Several factors including severe hyperthyroidism, large goiter size, persistent high thyrotropin receptor antibody (TRAb) levels, high anti-thyroid peroxidase 
(anti-TPO) antibody levels, the presence of orbitopathy, and smoking habit have been suggested as risk factors for relapse after discontinuation of ATDs [2, 3]. However, the results of the studies evaluating the association between gender and relapse of GD are generally conflicting. For instance, in a recent meta-analysis, male gender has not been found to be associated with relapse of GD after discontinuation of the ATDs, whereas in a study conducted by Allahbadia et al., after adjusting for the other confounding factors, relapse was significantly more common in male compared to female patients with GD, after discontinuation of the ATDs [4]. However, in studies assessing the association between gender and relapse of GD, the number of patients, particularly the number of male patients with GD is generally low, and therefore, the interpretation of the results is challenging. So, in this retrospective multicentre study, we examined the hypothesis that gender may affect the clinical response to treatment during ATD treatment, and relapse after discontinuation of the ATDs, in a large cohort of patients with GD, who were diagnosed, treated, and followed-up in six tertiary endocrinology referral centres.

\section{Material and methods}

\section{Patients}

This study included 717 (514 female and 203 male) patients with the first episode of GD from January 2008 to January 2019, in six endocrinology referral centres across Turkey. Patients $<18$ years old, pregnant patients, with ATD treatment $<12$ months, patients with a history of prior RAI treatment or thyroidectomy, and patients with major side effects of ATDs were not included in the study. Methimazole as a titration regimen was started in all patients, with an initial dose of 10-30 mg/day, and the ATD titration to the lowest dose (generally $2.5-5 \mathrm{mg} /$ day) was performed according to the follow-up free thyroxine (fT4) and free triiodothyronine (fT3) levels. However, patients with minor side effects of methimazole were switched to appropriate doses of propylthiouracil. All patients were classified as relapse, poorly controlled, or remission. The clinical and biochemical evaluation of the patients was performed at the initial visit before the start of ATDs, monthly until the restoration of thyroid functions, subsequently at three-month intervals during ATD treatment and dose titration, and at 3-6-month intervals after discontinuation of the ATDs.

Graves' disease, poorly controlled disease, and relapse were defined as previously reported [3]. In brief, GD was defined as clinical hyperthyroidism with or without extrathyroidal manifestations associated with suppressed or undetectable serum levels of thyroidstimulating hormone (TSH), elevated serum fT4 and/or fT3 levels, diffuse hypoechoic ultrasonographic appearance of the thyroid gland, high TRAb titres, or high uptake of $99 \mathrm{~m}$-Tc pertechnetate in association with clinical and biochemical findings in patients with TRAb levels within the normal reference ranges. The poorly controlled disease was defined as several episodes of hyperthyroidism followed by euthyroidism and rarely hypothyroidism or vice versa, occurring after titration of ATDs, during at least 12 months of follow-up, in a self-reported medication adherent patient [3]. Relapse was defined as overt clinical and biochemical hyperthyroidism in a euthyroid patient, after discontinuation of the lowest dose of ATDs. The follow-up time in patients with relapse and remission was recorded from the first day after the discontinuation of the
ATDs, and the beginning of the ATD treatment in patients with the poorly controlled disease, because treatment with ATDs was difficult to discontinue, even for several years in some of these patients. The study protocol was approved by the Local Ethics Committee.

\section{Clinical and laboratory data}

Clinical and laboratory data were obtained through the computer records of each centre. The following data of the patients at the first episode of GD were recorded: gender, age, serum TSH, fT3, fT4, TRAb, anti-TPO and anti-thyroglobulin (anti-TG) antibody levels, goiter size according to the WHO classification (0-III), smoking status, the presence of orbitopathy, the type of ATD used, date of start and discontinuation of ATDs, treatment duration, time of relapse, and application of definitive treatment, if any. Orbitopathy was classified according to Werner's criteria as absent (class $0-1$ ) or present (class 2-6: the presence of inflammatory signs, proptosis, extraocular muscle, and corneal involvement) [5].

Laboratory analysis to assess serum TSH, fT3, fT4, TRAb, anti-TPO, and anti-TG levels was performed during the first presentation of the patients in each study centre. Serum TSH, FT3, fT4, anti-TPO, and anti-TG levels were assessed by direct chemiluminescence immunoassay method in all centres (Cobas Elecsys, Roche Diagnostics, Manheim, Germany, and Siemens, ADVIA Centaur XP Immunoassay System, Tarrytown NY). Serum TRAb levels were assessed by chemiluminescence immunoassay, TRAb-Fast ELISA, radioimmunoassay, and radioreceptor assay methods (Cobas Elecsys, Roche Diagnostics, Manheim, Germany, Euroimmun AG Seekamp, Lübeck, Germany; anti-rTSH, RIA kits, Immunotech, Beckman Coulter, Prague, Czech Republic; and TRAK Dyno human, BRAHMS Germany) in different study centres. The normal laboratory reference values for TRAb, as were established by the manufacturers, were $<1.5 \mathrm{IU} / \mathrm{L}$ for the first two assay methods, $<$ $1.75 \mathrm{IU} / \mathrm{L}$ for the third assay method, and qualitatively as negative and positive in the last assay method.

\section{Statistical analysis}

The statistical analysis was performed using PASW Statistics 18 for Windows statistical software package (IBM Corp., Armonk, NY). The two-sample Wilcoxon rank-sum (Mann-Whitney) or Kruskal-Wallis tests were used for variables not fitting a normal (or parametric) distribution. In contingency tables for categorical variables, the Fisher or chi-squared tests were used. Cox proportional hazards regression models were used to estimate the association of gender and the other predetermined risk factor with the outcome of ATD treatment. In regression analysis, we used the same cut-off values for age, TRAb, goiter size, and fT4, which were previously determined by Vos et al. [6]. The Kaplan-Meier method was used to demonstrate the cumulative rate of remission according to gender during the first two years of follow-up. Missing data were not imputed and were left as lost. All hypothesis tests were two-sided, with a significance level of 0.05 .

\section{Results}

In the present study, during the mean follow-up time of $26.75 \pm 21.25$ months (between 1-120 months) after ATD withdrawal, $269(37.5 \%), 176(24.5 \%)$, and $272(37.9 \%)$ patients experienced a relapse, had a poorly controlled disease, and remained in remission, respectively. Relapse and poorly controlled disease were ensued in 185 $(36 \%)$ and $106(20.6 \%)$ of the females and $84(41.4 \%)$ and $70(34.5 \%)$ of the male patients, respectively $(\mathrm{p}=0.001$, $\chi^{2}$ test). During the follow-up period, $223(43.4 \%)$ of the females and only $49(24 \%)$ of the males remained in remission ( $p<0.001, \chi^{2}$ test). The time to relapse after 
Table 1. Clinical and biochemical characteristics of 717 patients with the first episode of Graves' disease (GD), as the whole group, and according to the gender of the patients

\begin{tabular}{|c|c|c|c|c|}
\hline Parameter & $\begin{array}{l}\text { All patients } \\
717(100 \%)\end{array}$ & $\begin{array}{c}\text { Female } \\
514(71.7 \%)\end{array}$ & $\begin{array}{l}\text { Male } \\
203(28.3 \%)\end{array}$ & $p$ value \\
\hline Age at diagnosis (years) & $42.27 \pm 13.6$ & $41.6 \pm 13.68$ & $43.9 \pm 13.31$ & \\
\hline$<40$ & $353(49.2)$ & $263(51)$ & $90(44.3)$ & \\
\hline$\geq 40$ & $364(50.8)$ & $251(49)$ & $113(55.7)$ & \\
\hline Goiter size & & & & 0.013 \\
\hline Grade $0(\%)$ & $44(6.1)$ & $32(6.2)$ & $12(5.9)$ & \\
\hline Grade I (\%) & $319(44.5)$ & $246(47.9)$ & $73(36)$ & \\
\hline Grade II (\%) & $274(38.2)$ & $185(36)$ & $89(43.8)$ & \\
\hline Grade III (\%) & $80(11.2)$ & $51(9.9)$ & $29(14.3)$ & \\
\hline $\operatorname{TRAb}[\mathrm{IU} / \mathrm{L}]^{*}$ & $11.96 \pm 11.74$ & $11.08 \pm 11.32$ & $12.21 \pm 12.13$ & 0.44 \\
\hline FT4 [pmol/L] & $44.46 \pm 22.09$ & $43.74 \pm 21.61$ & $46.28 \pm 23.21$ & 0.23 \\
\hline FT3 [pg/mL] & $10.95 \pm 6.68$ & $10.86 \pm 6.58$ & $11.17 \pm 6.97$ & 0.70 \\
\hline TSH [IU/L] & $0.009 \pm 0.012$ & $0.009 \pm 0.013$ & $0.007 \pm 0.007$ & 0.62 \\
\hline Anti-TPO [U/L] & $254 \pm 289$ & $36.7 \pm 282.1$ & $298 \pm 303$ & 0.08 \\
\hline Anti-TG [U/L] & $336 \pm 685.4$ & $330 \pm 643$ & $352.2 \pm 785.6$ & 0.67 \\
\hline Smoking status (Yes/No) & $284(39.6) / 433(60.4)$ & $169(32.9) / 335(65.2)$ & $115(56.7) / 87(42.9)$ & $<0.001$ \\
\hline Orbitopathy (Yes/No) & $203(28.3) / 514(71.7)$ & $133(25.9) / 380(73.9)$ & $70(34.5) / 132(65)$ & 0.053 \\
\hline Treatment duration* & $22.27 \pm 11.8$ & $22.08 \pm 11.1$ & $22.77 \pm 13.5$ & 0.88 \\
\hline Follow-up duration* & $26.75 \pm 21.25$ & $27.74 \pm 20.7$ & $24.25 \pm 22.48$ & 0.005 \\
\hline \multicolumn{5}{|l|}{ Treatment outcome } \\
\hline Remission (\%) & $272(37.9)$ & $223(43.4)$ & $49(24.1)$ & \\
\hline Relapse & $269(37.6)$ & $185(36)$ & $84(41.4)$ & $<0.001$ \\
\hline Poorly controlled disease & $176(24.5)$ & $106(20.6)$ & $70(34.5)$ & \\
\hline Non-remitting disease ${ }^{\dagger}$ & $445(62.1)$ & $291(56.6)$ & $154(75.9)$ & \\
\hline \multicolumn{5}{|l|}{ Final treatment } \\
\hline ATD (\%) & $167(23.3)$ & $121(23.5)$ & $46(22.6)$ & \\
\hline RAI treatment $(\%)$ & $129(18)$ & $72(14)$ & $57(28)$ & \\
\hline Total thyroidectomy (\%) & $149(20.8)$ & $98(19)$ & $51(25)$ & \\
\hline \multicolumn{5}{|c|}{$\begin{array}{l}\text { TRAb — thyrotropin receptor antibody; } \mathrm{fT} 4 \text { — free thyroxine; } \mathrm{fT} 3 \text { — free triiodothyronine; TSH — thyroid-stimulating hormone; anti-TPO — anti-thyroid peroxidase; } \\
\text { anti-TG — anti-thyroglobulin; ATD — antithyroid drugs; RAI — radioactive iodine, }{ }^{*} \text { months, }{ }^{,} \text {relapse + poorly controlled disease }\end{array}$} \\
\hline $\begin{array}{l}\text { The smoking status of } 11 \text { patien } \\
\text { The anti-TG antibody levels were } \\
33 \text { male) and were not included }\end{array}$ & $\begin{array}{l}\text { n. The orbitopathy status } \\
\text { or } 123 \text { patients. TRAb le } \\
\text { up analysis }\end{array}$ & $\begin{array}{l}\text { ents was unknown. The } A \\
\text { assessed qualitatively as }\end{array}$ & $\begin{array}{l}\text { antibody levels were not } \\
\text { and negative results in } 1\end{array}$ & $\begin{array}{l}\text { or } 102 \text { patients. } \\
\text { (84 female and }\end{array}$ \\
\hline
\end{tabular}

discontinuation of the ATDs was also significantly different between both genders. Among female patients, $28.2,24.1,38.8$, and $16.9 \%$ experienced a relapse during the first six months, between 7-12 months, between 13-24 months, and > 24 months after discontinuation of the ATDs, respectively, whereas among male patients, $49.5,24.2,14.7$, and $11.6 \%$ experienced a relapse in the same time periods, after discontinuation of the ATDs, respectively ( $\mathrm{p}=0.001, \chi^{2}$ test). As shown in Table 1 , male patients with GD also tended to have larger goiter size at presentation, as compared to female patients ( $\mathrm{p}=0.023)$. Other variables including TSH, fT3, fT4, TRAb, anti-TPO, and anti-TG antibodies were not dif- ferent between male and female patients (Tab. 1). Due to the quite similar clinical and biochemical features at presentation, the patients with relapse and the poorly controlled disease were combined in a single group as a non-remitting group in uni- and multivariate analysis. In univariate analysis, male gender, age $<40$ years, TRAb levels between 6-20 IU/mL, and goiter sizes II and III were significantly associated with the non-remitting GD (Tab. 2). However, although the effect of each parameter became smaller in multivariate analysis, male gender remained independently associated with non-remitting GD (Tab. 2). The cumulative rate of relapse according to the gender after discontinu- 
Table 2. The risk of relapse and poorly controlled disease (non-remitting disease) according to gender, age, TRAb level, and goiter size determined by uni-and multivariate analysis

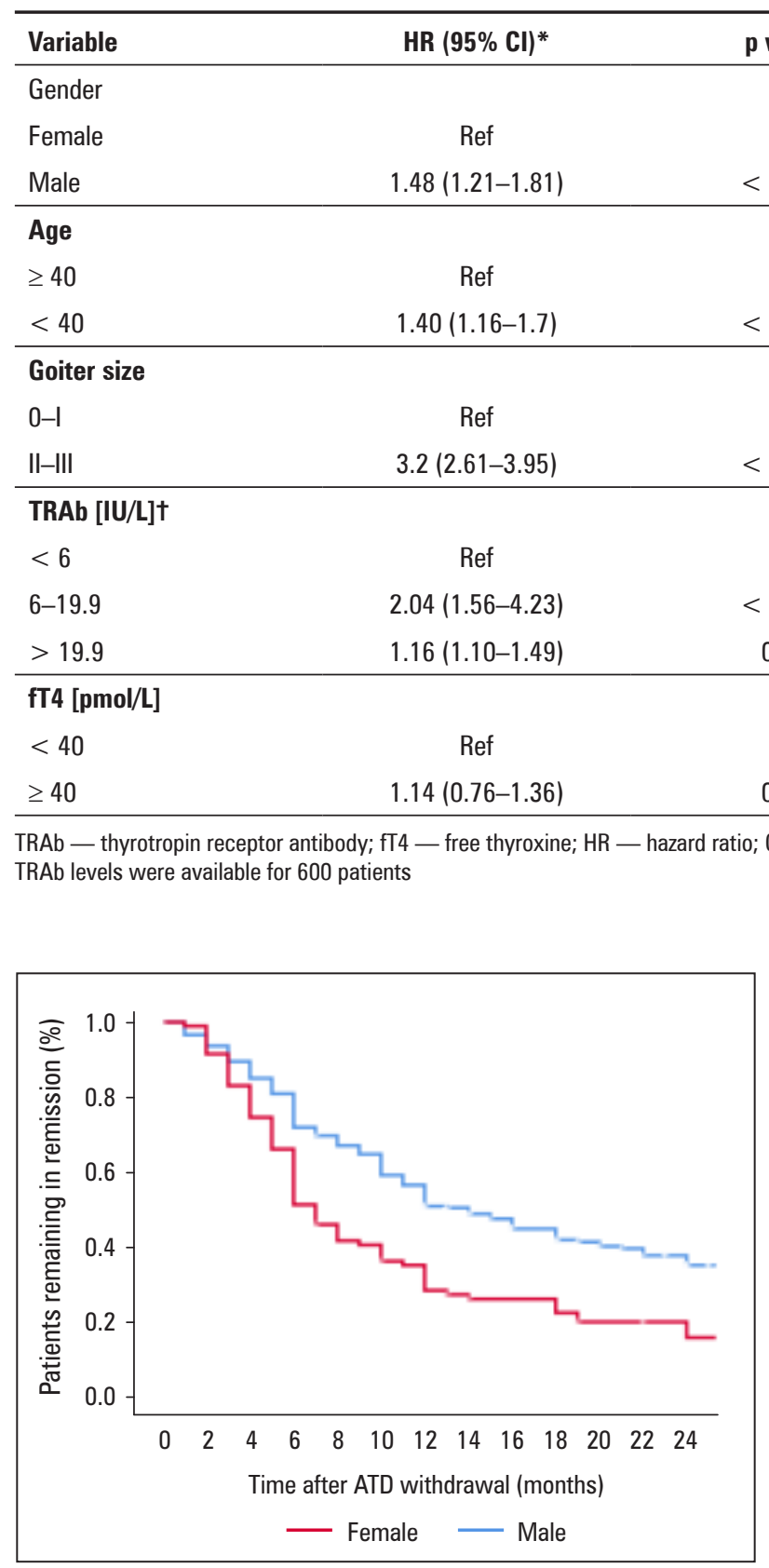

Figure 1. The cumulative rate of relapse according to gender after discontinuation of antithyroid drugs (ATDs), shown in a Kaplan-Meier curve

ation of the ATDs is shown in the Kaplan-Meier curve in Figure 1.

\section{Discussion}

In the present study, we evaluated the effect of gender on response to ATDs and the risk of relapse after discontinuation of ATDs. According to the results obtained from our study, male patients had a markedly worse outcome during and after ATD treatment, with a remission rate of only $24 \%$ compared with $43.4 \%$ for females.

An ideal treatment goal of GD would include the prompt control of the disease manifestations, restoration, and maintenance of the euthyroid state, minimal morbidity due to the disease itself, or adverse events resulting from treatment, as well as a reasonable cost [7]. Considering these treatment goals, treatment with ATDs is a reasonable treatment option in patients with GD. However, the relapse rate is high and occurs in $30-60 \%$ of cases after discontinuation of the ATDs [8]. On the other hand, although rare, ATDs are associated with some serious adverse reactions that could be life-threatening $[9,10]$. Therefore, the prediction of response to ATDs and risk factors for relapse before the start of ATDs are quite important. Several individual, as well as combined, risk factors have been suggested to predict the risk of relapse in patients with GD [2, 6-8, $11,12]$, but the results are far from ideal. Considering that females are more likely to develop autoimmune thyroid disease, including GD, compared to males [13] but males with GD may have more severe extrathyroidal manifestations, it has been suggested that males may be less responsive to ATDs and have a higher relapse rate [14]. However, as the results of the studies are conflicting and the level of evidence is low, the 2016 American Thyroid Association guidelines on diagnosis and management of hyperthyroidism state that there may be a higher likelihood of relapse after 
discontinuation of ATDs in males with GD, but do not make a definite recommendation on the choice of treatment between two genders [15]. Allahabdia et al. were among the first to assess the frequency of relapse between female and male patients with GD in the UK [4]. They found a higher rate of relapse in male compared to female patients with GD, after adjusting for possible confounders (OR $=2.80 ; 95 \% \mathrm{CI}$ : 1.31-5.98). They concluded that male patients with GD should be offered definitive treatment with radioiodine or surgery soon after presentation. However, in that study, TRAb levels at presentation, a potential risk factor for relapse after discontinuation of the ATDs, were not assessed. Our study results are in line with those reported by Allahabadia et al. In our study, $62 \%$ of the patients had non-remitting GD. Males, however, had a markedly worse outcome during and after ATD treatment, with a non-remitting GD rate of $76 \%$ compared with $56.6 \%$ for females $(\mathrm{p}=0.001)$. As demonstrated in Table 2, after adjusting for the well-known risk factors of relapse, male gender was independently associated with the risk of non-remitting disease [hazard ratio $(\mathrm{HR})=1.26,95 \%$ confidence interval $(\mathrm{CI}): 1.03-1.53$, $p=0.025]$. Furthermore, relapse during the first six months after discontinuation of the ATDs was more likely to occur in male patients with GD as compared to females. However, in a recent study carried-out by Diker-Cohen et al. in Israel, the relapse rate after discontinuation of the ATDs was not different between male and female patients with GD [16]. Nevertheless, in that study, other well-known risk factors for relapse, including younger age, fT4, and TRAb, were not associated with relapse of GD as well. The lack of association between these well-known risk factors and relapse in their study may be attributed to the high prevalence of iodine deficiency in Israel [17]; therefore, the results of their study may not apply to iodine-sufficient areas. In a recent meta-analysis, orbitopathy, smoking, larger goiter size and thyroid volume, fT4, fT3, and TRAb levels were significantly associated with relapse while gender and age did not show any association with relapse [2]. Recently, Magri et al. assessed the influence of gender on the outcome of treatment with ATDs in 294 patients with GD in Italy [18]. In that study, relapse after discontinuation of the ATDs was significantly more frequent in male compared to female patients with GD (59.1\% vs. $36.0 \%, \mathrm{p}=0.003)$. Although TRAb, fT3, and fT4 levels were not different between both genders, thyroid volume assessed by sonography was larger in males, and the frequency of autoimmune disease was more common among the relatives of males compared to females. They concluded that a larger goiter size and a strong genetic autoimmune background may explain the higher frequency of relapse in male patients with GD [18]. Our study results are in line with those reported by Magri et al. While the severity of hyperthyroidism at first diagnosis, as assessed by fT3 and fT4 levels, was not different between male and female patients, the goiter size assessed by manual palpation was significantly lager in male patients (Tab. 1).

The association between relapse of GD and goiter size has been demonstrated in several studies [2, 3, 6], but the reason for the larger goiter size in male compared to female patients with GD is not clear so far and should be investigated in further studies. Although the goiter size in healthy euthyroid males is larger than females due to the higher body weight [19], as shown in Table 1, grade II and III goiter were also present in $46 \%$ of the females in our study. We think that moderate iodine insufficiency in our country may have contributed to the larger goiter sizes detected in our study participants [20]. Another factor that may have contributed to a higher frequency of relapse in male patients in our study is the higher frequency of smoking habit among males. Studies have shown a significantly higher frequency of relapse of GD in tobacco smokers, particularly in male patients $[2,21]$. Although statistically did not reach significance, smoking habit was also more frequent among male patients in the study of Magri et al. $(42.5 \%$ vs. $32.5 \%)$ [18].

One of the main differences between our study and the studies cited above $[4,16,18]$ is that we included a subgroup of patients with poorly controlled disease. These patients share similar clinical and biochemical features with patients who experience relapse [3]. As demonstrated in Table 1, the poorly controlled disease was also more frequent in male compared to female patients $(p<0.001)$. This result suggests that male gender is also associated with a higher frequency of poorly controlled disease as well as relapse. Therefore, the gender of the patient should be taken into account when considering treatment for GD.

Our study has several limitations. The first, main limitation is the retrospective design, which makes our study subject to reporting bias. Second, TRAb levels were assessed in 117 patients by qualitative method; therefore, the TRAb levels of these patients could not be included in uni- and multivariate analysis, as well as in between groups analysis. Third, orbitopathy was not recorded according to the severity and activity as recommended by the recently published guidelines [22]. Fourth, goiter size was only assessed by manual palpation rather than thyroid ultrasonography. On the other hand, the strength of our study derives from the inclusion of a large number of patients, particularly large number of male patients with GD, with relatively long-term follow-up data. 


\section{Conclusions}

According to the present study results, relapse and poorly controlled disease during treatment with ATDs were significantly more common in male compared to female patients with GD. GD in male patients tended to relapse earlier compared to females. The higher frequency of relapse and poorly controlled disease in male patients might be attributed to the larger goiter sizes as well as a higher frequency of smoking in male patients. Nevertheless, the exact cause and whether the genetic background of male patients plays a role in the higher frequency of relapse as well as a poorly controlled disease should be determined in further studies. However, along with other well-known risk factors for relapse, the gender of the patients should be taken into consideration when deciding treatment for GD.

\section{Declaration of interest}

No potential conflict of interest was reported by the authors.

\section{References}

1. Nyström HF, Jansson S, Berg G. Incidence rate and clinical features of hyperthyroidism in a long-term iodine sufficient area of Sweden (Gothenburg) 2003-2005. Clin Endocrinol (Oxf). 2013; 78(5): 768-776, doi: 10.1111/cen.12060, indexed in Pubmed: 23421407.

2. Struja T, Fehlberg H, Kutz A, et al. Can we predict relapse in Graves' disease? Results from a systematic review and meta-analysis. Eur J Endocrinol. 2017; 176(1): 87-97, doi: 10.1530/EJE-16-0725, indexed in Pubmed: 27780830.

3. Zuhur SS, Elbuken G, Yildiz I, et al. External Validation of the GREAT Score in Turkish Patients with Graves' Hyperthyroidism Treated with the Titration Regimen Method of Antithyroid Drugs: A Multicenter Study. Horm Metab Res. 2019; 51(10): 627-633, doi: 10.1055/a-0974-3991, indexed in Pubmed: 31499558.

4. Allahabdia A, Daykin J, Holder RL, et al. Age and Gender Predict the Outcome of Treatment for Graves' Hyperthyroidism. J Clin Endocrinol Metab. 2000; 85(3): 1038-1042, doi: 10.1210/jcem.85.3.6430, indexed in Pubmed: 10720036.

5. Werner SC. Modification of the classification of the eye changes of Graves' disease: recommendations of the Ad Hoc Committee of the American Thyroid Association. J Clin Endocrinol Metab. 1977; 44(1): 203-204, doi: 10.1210/jcem-44-1-203, indexed in Pubmed: 576230.

6. Vos XG, Endert E, Zwinderman AH, et al. Predicting the Risk of Recurrence Before the Start of Antithyroid Drug Therapy in Patients
With Graves' Hyperthyroidism. J Clin Endocrinol Metab. 2016; 101(4): 1381-1389, doi: 10.1210/jc.2015-3644, indexed in Pubmed: 26863422.

7. Alsanea $\mathrm{O}$, Clark OH. Treatment of Graves' disease: the advantages of surgery. Endocrinol Metab Clin North Am. 2000; 29(2): 321-337, doi: 10.1016/s0889-8529(05)70134-1, indexed in Pubmed: 10874532.

8. Struja T, Kaeslin M, Boesiger F, et al. External validation of the GREAT score to predict relapse risk in Graves' disease: results from a multicenter, retrospective study with 741 patients. Eur J Endocrinol. 2017; 176(4): 413-419, doi: 10.1530/EJE-16-0986, indexed in Pubmed: 28100628.

9. Kahaly GJ, Bartalena L, Hegedüs L, et al. 2018 European Thyroid Association Guideline for the Management of Graves' Hyperthyroidism. Eur Thyroid J. 2018; 7(4): 167-186, doi: 10.1159/000490384, indexed in Pubmed: 30283735.

10. Cooper DS. Antithyroid drugs. N Engl J Med. 2005; 352(9): 905-917, doi: 10.1056/NEJMra042972, indexed in Pubmed: 15745981.

11. Sundaresh V, Brito JP, Wang Z, et al. Comparative effectiveness of therapies for Graves' hyperthyroidism: a systematic review and network meta-analysis. J Clin Endocrinol Metab. 2013; 98(9): 3671-3677, doi: 10.1210/jc.2013-1954, indexed in Pubmed: 23824415.

12. Piantanida E, Lai A, Sassi L, et al. Outcome Prediction of Treatment of Graves' Hyperthyroidism with Antithyroid Drugs. Horm Metab Res. 2015; 47(10): 767-772, doi: 10.1055/s-0035-1555759, indexed in Pubmed: 26197855.

13. Desai MK, Brinton RD. Autoimmune Disease in Women: Endocrine Transition and Risk Across the Lifespan. Front Endocrinol (Lausanne). 2019; 10: 265, doi: 10.3389/fendo.2019.00265, indexed in Pubmed: 31110493.

14. Fatourechi V. Is sex a predictor of outcome of antithyroid therapy of Graves hyperthyroidism. Endocr Pract. 2019; 25(1): 109-111, doi: 10.4158/EP-2018-0523, indexed in Pubmed: 30742572.

15. Ross DS, Burch HB, Cooper DS, et al. 2016 American Thyroid Association Guidelines for Diagnosis and Management of Hyperthyroidism and Other Causes of Thyrotoxicosis. Thyroid. 2016; 26(10): 1343-1421, doi: 10.1089/thy.2016.0229, indexed in Pubmed: 27521067.

16. Diker-Cohen T, Duskin-Bitan H, Shimon I, et al. Disease presentation and remission rate in Graves disease treated with antithyroid drugs: Is gender really a factor? Endocr Pract. 2019; 25(1): 43-50, doi: 10.4158/EP-2018-0365, indexed in Pubmed: 30383487.

17. Ovadia YS, Arbelle JE, Gefel D, et al. First Israeli National Iodine Survey Demonstrates Iodine Deficiency Among School-Aged Children and Pregnant Women. Thyroid. 2017; 27(8): 1083-1091, doi: 10.1089/thy.2017.0251, indexed in Pubmed: 28657479.

18. Magri F, Zerbini F, Gaiti M, et al. Gender influences the clinical presentation and long-term outcome of Graves' disease. Endocr Pract. 2016; 22(11): 1336-1342, doi: 10.4158/EP161350.OR, indexed in Pubmed: 27540885.

19. Berghout A, Wiersinga WM, Smits NJ, et al. Determinants of thyroid volume as measured by ultrasonography in healthy adults in a non-iodine deficient area. Clin Endocrinol (Oxf). 1987; 26(3): 273-280, doi: 10.1111/j.1365-2265.1987.tb00784.x, indexed in Pubmed: 3308184.

20. Erdoğan MF, Ağbaht K, Altunsu T, et al. Current iodine status in Turkey. J Endocrinol Invest. 2009; 32(7): 617-622, doi: 10.1007/BF03346519, indexed in Pubmed: 19564718.

21. Kimball LE, Kulinskaya E, Brown B, et al. Does smoking increase relapse rates in Graves' disease? J Endocrinol Invest. 2002; 25(2): 152-157, doi: 10.1007/BF03343979, indexed in Pubmed: 11929086.

22. Bartalena L, Baldeschi L, Boboridis K, et al. European Group on Graves' Orbitopathy (EUGOGO). The 2016 European Thyroid Association/European Group on Graves' Orbitopathy Guidelines for the Management of Graves' Orbitopathy. Eur Thyroid J. 2016; 5(1): 9-26, doi: 10.1159/000443828, indexed in Pubmed: 27099835. 